\title{
The Impact of High-Dose Vitamin C on Blood Glucose Testing in ${ }^{18} \mathrm{~F}$-FDG PET Imaging
}

\author{
Rebekah L. Bahr, RTNM ${ }^{1}$, and Don C. Wilson, FRCPC ${ }^{2}$ \\ ${ }^{1}$ Functional Imaging Program, BC Cancer Agency, Vancouver, British Columbia, Canada; and ${ }^{2}$ BC Cancer Agency, Vancouver, \\ British Columbia, Canada
}

\begin{abstract}
Complementary and alternative therapies in addition to standard oncology protocols are commonly sought by cancer patients; however, few patients disclose their complementary treatments to their cancer care team. A lack of communication may result in unforeseen side effects and the potential for some alternative therapies to interfere with or inhibit conventional treatment. High-dose vitamin C therapy, in particular, may lead to an inability to measure a patient's blood glucose level before ${ }^{18} \mathrm{~F}-\mathrm{FDG}$ injection for PET/CT scanning. We report a case of a 52-y-old woman referred for ${ }^{18} \mathrm{~F}-\mathrm{FDG}$ PET/CT to evaluate the extent of recurrent colorectal cancer. The PET/CT scan immediately followed a single intravenous dose of $25 \mathrm{~g}$ of ascorbic acid from her naturopath. A glucometer that applies the glucose oxidase method for measuring fasting blood glucose was used, for which high doses of vitamin $\mathrm{C}$ are listed as a contraindication. The high concentration of ascorbic acid in the patient's blood sample interfered with the chemical reaction on the glucose strip, and therefore no blood glucose measurement could be attained. With more patients receiving alternative and complementary cancer therapies, it is important to know what the implications of orthomolecular therapy might be on routine blood glucose testing for ${ }^{18} \mathrm{~F}-\mathrm{FDG}$ PET scans. ${ }^{18} \mathrm{~F}-$ FDG is in direct competition with glucose; therefore, elevated blood glucose levels will cause a decrease in ${ }^{18} \mathrm{~F}-\mathrm{FDG}$ absorption and may lead to a false-negative scan.
\end{abstract}

Key Words: vitamin C; ascorbic acid; blood glucose; PET

J Nucl Med Technol 2015; 43:70-71

DOI: 10.2967/jnmt.114.140335

A

Iternative therapies combined with standard oncology protocols are commonly sought by cancer patients; however, few patients disclose their complementary treatments to their care team. A lack of communication may result in unforeseen side effects and can potentially interfere with conventional treatment. Intravenous high-dose vitamin C (ascorbic acid) therapy, in particular, may lead to an in-

Received Mar. 14, 2014; revision accepted Apr. 30, 2014

For correspondence or reprints contact: Don C. Wilson, BC Cancer Agency, Vancouver Centre, 600 W. 10th Ave., Vancouver, BC, Canada, V5Z4E6.

E-mail: dowilson@bccancer.bc.ca

Published online Aug. 7, 2014.

COPYRIGHT (c) 2015 by the Society of Nuclear Medicine and Molecular Imaging, Inc. ability to measure a patient's blood glucose level before ${ }^{18} \mathrm{~F}-\mathrm{FDG}$ injection for PET/CT scanning.

\section{CASE STUDY}

A 52-y-old woman was referred for an ${ }^{18} \mathrm{~F}$-FDG PET/CT scan to determine the extent of recurrent colorectal carcinoma. Fasting blood glucose level was measured before ${ }^{18} \mathrm{~F}-\mathrm{FDG}$ injection by the drawing of a venous blood sample from an intravenous catheter. As per manufacturer guidelines, a drop of blood was placed on the glucose test strip (SureStep Pro; Lifescan). However, the drop of blood did not produce the anticipated reaction in which a blue color appears on the strip. The procedure was repeated with a new sample of blood and a new strip and was also unsuccessful.

Several blood samples were then obtained both from the patient's intravenous line and from capillary samples from finger pokes, none of which reacted with any glucose strip. Six separate glucose strips were used from 3 different containers stored in various locations and 2 different lot numbers of strips.

On further questioning, it was found that $2 \mathrm{~h}$ before her PET/CT appointment, the patient had undergone an intravenous infusion of $25,000 \mathrm{mg}$ of vitamin C. The package insert from the SureStep Pro glucometer test strips informs that vitamin $\mathrm{C}$ is an interfering substance but has no significant effect on blood glucose readings for physiologic vitamin $\mathrm{C}$ concentrations of up to $0.2 \mathrm{mmol} / \mathrm{L}$ (1). No other interfering substances were identified for this patient. No alternate means for testing blood glucose were accessible, but the patient was not diabetic and had followed a proper fasting protocol; therefore, ${ }^{18} \mathrm{~F}$-FDG was administered and the PET/CT scan was performed. On image review, the scan results and ${ }^{18}$ F-FDG biodistribution did not seem to be compromised despite the vitamin $\mathrm{C}$ infusion and inability to measure the patient's blood glucose level.

\section{DISCUSSION}

To measure blood glucose level using the Surestep Flexx Bedside Glucometer (Lifescan), a drop of blood is placed on the glucose strip and the blood reacts with glucose oxidase, triggering oxidation of glucose in the blood sample. Gluconic acid and hydrogen peroxidase are produced as a result of this reaction. Peroxidase on the test strip then causes the hydrogen peroxide to react with dyes to produce a blue color in the presence of oxygen. The 
glucometer is calibrated to interpret the patient's blood glucose level, which is proportional to the intensity of blue produced on the sample glucose strip.

Inaccurate readings of blood glucose occur when high plasma concentrations of vitamin $\mathrm{C}$ interfere with chromagen oxygenation on the blood glucose strip, and no blue color appears (2). Typical doses of vitamin $\mathrm{C}$ would not cause interference during blood glucose testing, but large doses of intravenously administered ascorbic acid (50-100 $\mathrm{mg}$ ) bypass absorptive mechanisms, producing high plasma concentrations of about $14,000 \mu \mathrm{mol} / \mathrm{L}(3)$.

A pioneer of megavitamin therapy and Nobel Prize winner, Linus Pauling advocated vitamin $\mathrm{C}$ as a prooxidant because it generates hydrogen peroxide $\left(\mathrm{H}_{2} \mathrm{O}_{2}\right)$ in extracellular spaces. Hydrogen peroxide present in blood is toxic to cancer cells but not normal cells (4); thus, patients believe it will destroy cancer cells and free radicals such as those produced in the presence of ionizing radiation.

\section{CONCLUSION}

The inability to measure this patient's blood glucose level with the glucometer test strip was most likely caused by interference from a high dose of vitamin $\mathrm{C}$ infused just before her PET/CT appointment. Having an accurate glucose reading before ${ }^{18} \mathrm{~F}-\mathrm{FDG}$ injection is extremely important to ensure accurate ${ }^{18} \mathrm{~F}-\mathrm{FDG}$ PET/CT scanning results. Technologists should understand the mechanics of the glucometer in use and know the limitations of glucose testing. Patients should be encouraged to disclose any alternative treatments to their care team.

\section{DISCLOSURE}

No potential conflict of interest relevant to this article was reported.

\section{REFERENCES}

1. SureStep Pro Test Strips [package insert]. Milipitas, CA: Lifescan; 2003.

2. Tang Z, Du X, Louie R, Kost G. Effects of drugs on glucose measurements with handheld glucose meters and a portable glucose analyzer. Am J Clin Pathol. 2000;113:75-86.

3. Padayatty SJ, Riordan H, Hewitt S, Katz A, Hoffer L, Levine M. Intravenously administered vitamin $\mathrm{C}$ as cancer therapy: three cases. CMAJ. 2006;174:937942.

4. Chen Q, Espey M, Krishna M, et al. Pharmacologic ascorbic acid concentrations selectively kill cancer cells: action as a pro-drug to deliver hydrogen peroxide to tissues. Proc Natl Acad Sci USA. 2005;102:13604-13609. 